WORLD COMPLEXITY SCIENCE ACADEMY JOURNAL| Vol. 1 Iss. 3, 21 | Winter 2020

\title{
EVOLUTION OF LIFE: A CONDITIONAL CAUSALITY PHENOMENON
}

\author{
Chi Kuo Mao, ${ }^{1}$ Eric M. Chang ${ }^{1 *}$
}

\author{
${ }^{1}$ Institute of Business and Management, National Chiao Tung University, Taipei, Taiwan \\ * Correspondent author: Eric M. Chang - eric.bm04g@nctu.edu.tw
}

Received:13.10.2020; Accepted: 19.11.2020; Published: 20.12.2020

DOI: https://doi.org/10.46473/WCSAJ27240606/20-12-2020-0021

Category: Research paper

\begin{abstract}
Inspired by Ilya Prigogine's dissipative structure theory proposed in the 1970s, the authors of this paper, based on an examination of several selected pivotal incidents in the evolutionary history of life on Earth, have arrived at two conclusions, namely that all incidents on Earth follow a common principle of Conditional Causality, and that they repeat through Cyclic Adaptation. This common principle of Conditional Causality, based on evidence we have gathered from other secondary sources, apparently governs all incidents, and this principle is also the underlying principle seen in the behaviours of the dissipative structure. The Conditional Causality principle, as we have observed, is a principle of the life system itself the principle not only explains the creation, existence, and evolutionary process of a life system, but also provides us with a basis for the development of a new open system paradigm to supplement, if not replace, the current Newtonian mechanistic system paradigm.
\end{abstract}

Keywords: Dissipative Structure Theory, Conditional Causality, Resultant Effect, Cyclic Adaptability, Symbiont

\section{Introduction}

Dissipative structure theory (DST) was proposed by Ilya Prigogine, a Nobel laureate in chemistry in 1977. DST is based on thermodynamics and is used to study the self-organizing behaviours of nonequilibrium systems. Prigogine has outlined the fundamental characteristics of the dissipative structure as follows:

1. Order and organisation can actually arise "spontaneously" out of disorder and chaos through a process of "self-organisation" (Prigogine and Nicolis, 1977: forewod).

2. New dynamic states of matter may originate, states that reflect the interaction of a given system with its surroundings (Prigogine and Stengers, 1984: 12).

3. Nonequilibrium may be a source of order, and irreversible processes may lead to a new type of dynamic states of matter (Prigogine, 1978: 1). 
DST represents the principle of interaction between an open system comprising selforganizing elements and the system's external environment (Dooley and Van de Ven, 1999; Melo et al., 2019; Ruzzeddu and Roblek, 2020). Specifically, the principle is as follows:

1. When a significant change occurs in the external environment to which the system belongs, the internal elements of the system will spontaneously produce an ordered dissipative structure from a disordered structure through a self-organizing process.

2. This dissipative structure will change again along with the environment's new changes, and a new dissipative structure will spontaneously emerge once more to adapt to the new environment.

These interpretations indicate the existence of a cause-condition relationship between the system and the environment. The term "cause" here refers to the system, and "condition" the environment. Accordingly, the convergence of the cause and condition produces a certain effect, in which a self-organizing force drives the aforementioned Conditional Causality process (Mao, 2018). However, Prigogine has only drawn examples from thermodynamics and chemistry (Cramer,1993). The objective of this paper is to analyse some relatively remarkable evolutionary events from Earth's history to examine whether DST applies to all life systems. For this paper, several pivotal incidents have been selected from the evolutionary history of life on Earth to test the authors' hypothesis regarding Conditional Causality and Cyclic Adaptation.

Fig. 1 depicts how a Conditional Causality process functions. When the external condition (the environment) changes significantly, a self-organizing mechanism of the internal cause (the system) will be activated to prepare the system itself to adapt to the new environment; eventually, a certain resultant effect (a new system or a new environment. Hereinafter simplified as "result") emerges.

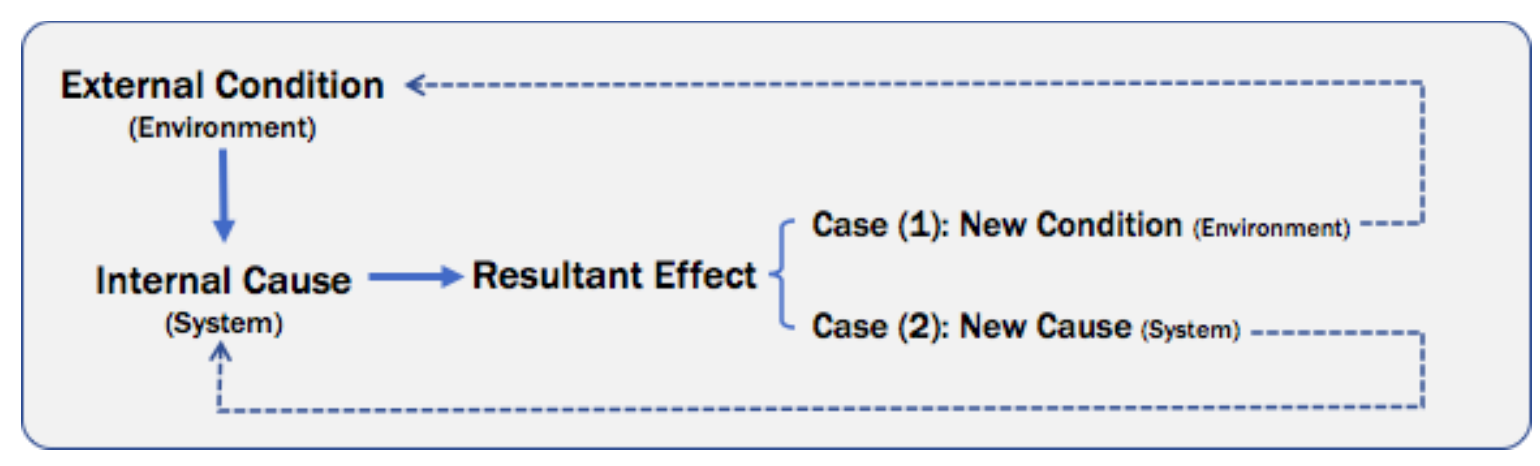

Figure 1. The mechanism of the Conditional Causality process

\section{Hypothesis and Methodology}

Specifically, the sub-hypotheses of the Conditional Causality principle proposed in this paper are as follows:

H1. As far as DST is concerned, the evolution of a living being is a result of the interaction between the living being and the conditions of the living being's environment. In this paper, where a living being is treated as an internal cause, the principle governing the evolutionary process of the living being is called Conditional Causality. 
H2. For any given living being (the internal cause), the evolutionary process is normally activated by a drastic change in the external environmental conditions. When the evolutionary process begins, the living being will then start an adaptive process of selforganization to transform itself to suit the new environment.

H3. Normally, the result of the interaction between the living being and the external conditions is the appearance of a new version of the previous living being. As shown by Case 2 with a dotted line in Fig. 1, any living beings will be repeatedly subject to the cyclic effects of drastic external changes and, consequently, exhibit cyclic adaptability to ensure their vitality in the new environment, as indicated by the lower dotted loop in Fig. 1.

H4. On certain occasions, the result could be a newly emerged environment rather than a new version of the previous living being - this refers to Case 1 shown in Fig. 1.

\section{Selected Evolutionary Events and Analyses}

A review of academic research on the origin of life would conclude that there was no ocean, oxygen, or life on Earth 4.6 billion years ago. There are three milestones in the evolution of life in the early stages: (1) The emergence of RNA as the primary living substance in prokaryotes, followed by DNA and enzymes, which established the important initial state of life forms 4 billion years ago (De Duve, 1995; Margulis and Sagan, 1997; Lane, 2010). (2) The appearance of cyanobacteria, a type of prokaryote with the capacity to produce carbohydrates through photosynthesis that emerged 3.4 billion years ago under sufficient sunlight (Margulis et al., 2006). (3) The Great Oxygenation Event (GOE) which occurred 2.6 billion years ago and caused major extinction of the anaerobic prokaryotes that had survived through fermentation (Dole, 1965; Margulis and Sagan, 1997).

\subsection{Background Events}

Both the GOE and the endosymbiotic life forms that resulted thereafter provided the contextual background and basis for the subsequent evolution of life as seen in the history of evolution.

\subsubsection{GOE}

Before the GOE, there was practically no oxygen on Earth, but sunlight was sufficient. Most of the organisms were heterotrophic, except cyanobacteria and a few other organisms that were autotrophic. Photosynthesis of the cyanobacteria incrementally released oxygen into the atmosphere until oxygen occupied one-fifth of the atmosphere (Dole, 1965; Margulis and Sagan, 1997). The GOE is the result of Conditional Causality and not a new internal causebut a new external condition.

\subsubsection{Endosymbiosis}

Oxygen was poisonous for most of the organisms that had lived without oxygen; therefore, the GOE killed most of them. Although most life struggled, a type of small bacteria survived by becoming aerobic. Certain prokaryotes capitalised on the ex istence of these bacteria and evolved into eukaryotes through endosymbiosis 1.8 billion years ago. Under pressure from the GOE, some anaerobic prokaryotes phagocytised aerobic bacteria and became eukaryotic (Margulis et al., 2006). Characteristics of the endosymbiosis were as follows: First, the host 
cell and parasitic cell maintained a symbiotic coexistence, and their DNA continued to biologically evolve independently. Second, the engulfed aerobic bacteria became the mitochondria that provided the energy required by the endosymbiont. Third, the archaea protected the aerobic bacteria from external harm. (Margulis et al., 2006; Zachar and Szathmáry, 2017; Zachar et al., 2018; Imachi et al., 2020).

The prokaryotes evolved into eukaryotes through endosymbiosis so as to survive the GOE crisis. This evolution is clear evidence of the existence of Conditional Causality. Furthermore, the eukaryotes formed through endosymbiosis then became the fundamental structural module for all multicellular living systems (i.e., eukaryotic cells that evolved through endosymbiosis from individual single-celled prokaryotes became the origin of multi-celled organisms). Eukaryotes are not only the fundamental constituent elements of animals and plants but also the origin of eukaryotic cellular complexity.

The following section examines selected major events in the evolution of life on Earth and describes interactions among relevant causes, conditions, and results. The purpose of this examination is to demonstrate the validity of the Conditional Causality and the Cyclic Adaptation hypotheses presented above as $\mathrm{H} 1$ through $\mathrm{H} 4$.

\subsection{Evolution of Plants}

\subsubsection{Origin of plants}

I. Condition-After the GOE, oxygen concentration in the atmosphere rose significantly.

II. Cause-Oxygen was harmful and caused most organisms to die. Some anaerobic prokaryotes, photosynthetic cyanobacteria, and aerobic bacteria adapted to the postGOE environment through endosymbiosis in order to survive.

III. Result-Prokaryotes that undertook the endosymbiotic process mentioned above, evolved into eukaryotes, some of which diversified into plant cells. Since endosymbiotic relationship increased the survival rate of eukaryotes, this implies that, for prokaryotes, forming a symbiotic partnership with the engulfed bacteria was more favourable than digesting them, under the condition that sunlight is sufficient (Sagan, 1967; Margulis and Sagan, 1997; Zachar et al., 2018).

\subsubsection{Development of bryophytes}

I. Condition-In the late Silurian age (440-410 million years ago), a collision of continents resulted in the Euramerica landmass. Consequently, Earth's appearance changed significantly.

II. Cause-Some algae grew in the intertidal zone and remained on the land after the tide receded.

III. Result - Some of the algae in the intertidal zone acquired genes from soil bacteria that made them resistant to desiccation, and enabled them to fix nitrogen (Cheng et al., 2019). This algae evolved into bryophytes, which grew on land and developed cuticles that prevented water from dissipating when the tide ebbed. They also developed stomata for heat dissipation (Lane, 2010). 


\subsubsection{Development of vascular plants}

I. Condition - Continental uplifts resulted in large stretches of intertidal zones, which contained more resources essential for life than the ocean.

II. Cause-Bryophytes flourished on land near water and gradually evolved and moved inland (De Duve, 1995).

III. Result - To access more sunlight for photosynthesis, plants gradually evolved to grow taller. Thus, plants developed vascular bundles that can transport and store water and nutrients to support their structure. Bryophytes evolved into ferns comprising roots, stems, and leaves as organs. Ferns were the dominant plants in the Devonian period (400-360 million years ago) (Lane, 2010).

\subsubsection{Rise of seed plants}

I. Condition - In the Permian period (300-250 million years ago), Pangaea was a unified continent and was the product of a continental collision (De Duve, 1995).

II. Cause-Ferns required on humid environments for survival because their spores (sporophytes) must be fertilised in water. This constrained their expansion inland.

III. Result - The Pteridospermatophyta (or seed ferns) developed pollen sacs and seeds on their leaves (Lane, 2010). Seed plants emerged and were no longer constrained by their dependence on water for fertilisation. Consequently, they could expand inland (De Duve, 1995; Margulis and Sagan, 1997; Lane, 2010).

\subsection{Evolution of Animals}

\subsubsection{Origin of animals (eukaryotes)}

I. Condition-The GOE led to the emergence of animal cells, it is similar to the emergence of plant cells.

II. Cause- Prokaryotes were anaerobic and some bacteria had oxidative abilities. Both were independent organisms at the beginning.

III. Result-While anaerobic prokaryotes could not survive in oxygenated air, they could provide protection for the parasitic bacteria. The parasitic bacteria, , on the other hand, have adapted to oxygenated environments. Therefore, the prokaryotes acquired the aerobic bacteria and overcame their anaerobic limitation. This in turn enabled the parasitic bacteria to share the resultant adaptive compound with the prokaryotes, the host cells., Ultimately, they depended on each other for survival, and evolved into animal eukaryotes. The bacteria that is contained in eukaryotes maintained their own DNA and evolved into mitochondria (Sagan, 1967; Zachar et al., 2018).

\subsubsection{Origin of amphibians}

I. Condition-The intertidal zone was rich with nutrients and other resources, attracting animals to inhabit and propagate. Several animals moved onto land. Additionally, the ozone layer gradually developed properties to shield living organisms from harmful UV (De Duve, 1995; Margulis and Sagan, 1997). 
II. Cause-The Sarcopterygii (lobe-finned fish) had an anterior fin composed of muscle, and two ventral fins, all of which became the prototypal erect limbs (Lane, 2010). Due to the needs to find sustenance and escape predators, they lived in the intertidal zone.

III. Result - Between Silurian and Devonian periods (420-390 million years ago), the Sarcopterygii evolved into amphibians and walked on legs; they also started to breathe using lungs. However, they still had to live near water (De Duve, 1995; Lane, 2010).

\subsubsection{Origin of reptiles}

I. Condition - In the Carboniferous period (359-299 million years ago), the richness of terrestrial plants led to flourishing ecosystem on land (Lane, 2010).

II. Cause-Amphibians developed lungs and limbs for living ashore.

III. Result - They evolved into reptiles and developed bony plates to prevent the loss of moisture from their bodies. They also developed amnions, which provided a protective environment for their gestating embryos. Reptiles then became the dominant land animals (De Duve, 1995; Margulis and Sagan, 1997; Lane, 2010).

\subsubsection{Rise of mammals}

I. Condition-Researchers estimated that the Cretaceous-Paleogene extinction event (66 million years ago) was caused by a meteorite impact followed by a period of volcanism. Dust dispersed into the atmosphere, causing long-term effects on the climate and ecosystem. Large animals such as dinosaurs became extinct, and only small animals managed to survive (Lane, 2010).

II. Cause-Several characteristics of the order Therapsida are consistent with that of mammals, i.e., erect limbs, warm blood, and fur conducive to the preservation of body heat. These characteristics facilitated their adaptation to the new environment (Margulis and Sagan, 1997).

III. Result-Smaller animals survived because of the sudden decrease in predators, making it easier to forage. Viviparous birth and breastfeeding not only ensured survivability but also helped with offspring development.

\section{Conclusions}

\subsection{Synthesis of Findings}

Table 1 presents results obtained from the testing of the four sub-hypotheses of selected events in life's evolution. Clearly, all the mentioned incidents were governed by a common principle of Conditional Causality; that is, when the external environment changes drastically, the internal cause of the life system would demonstrate resilience (i.e. 'life finds its way') to survive, and a result (a new system) sustainable to the new environment would emerge. When the next drastic environmental change occurs, the new system would undergo another adaptive process and evolve again to strive for survival. The phenomenon of Cyclic Adaption is also clearly observed from the consecutive occurrence of the selected incidents. 
Table 1. Results of hypothesis testing ( $\circ$ : confirmed, -: nonapplicable)

\begin{tabular}{|l|c|c|c|c|}
\hline Events & H1 & H2 & H3 & H4 \\
\hline GOE & 0 & 0 & - & O \\
\hline Endosymbiosis & 0 & 0 & - & - \\
\hline The Origin of Eukaryotic Plant Cells & 0 & 0 & 0 & - \\
\hline The Emerging of land based Vascular Plants & 0 & 0 & 0 & - \\
\hline The Emerging of Seed Plants & 0 & 0 & 0 & - \\
\hline The Origin of Eukaryotic animal cells & 0 & 0 & 0 & - \\
\hline The Origin of Amphibians & 0 & 0 & 0 & - \\
\hline The Emerging of Reptiles & 0 & 0 & 0 & - \\
\hline The Emerging of Mammals & 0 & 0 & 0 & - \\
\hline
\end{tabular}

The timeline of the crucial life evolutionary incidents selected in this paper is depicted in Fig. 2. It is notable that the cycle time of the major incidents became shorter in later periods. Fig. 2 also indicates that the life evolutionary history follows punctuated equilibrium theory (Gould and Eldredge, 1972). 


\section{$102(3)(4)(5)(7) 898$}

(1) The birth of Earth 4.6 billion years ago

(2) Cyanobacteria originated 3.4 billion years ago

(3) GOE 2.6 billion years ago

(4) Eukaryote originated 1.8 billion years ago

(5) Bryophyte ashore 440 million years ago
(6) Amphibians originated 420 million years ago

(7) Vascular plants ashore 400 million years ago

(8) Reptiles originated 320 million years ago

(9) Seed plants raised 300 million years ago

(10) Mammals raised 66 million years ago

Figure 2. The Timeline of Life Evolution Major Incidents

\subsection{Theoretical Implications}

In this paper, based on analysis of several selected pivotal incidents in the evolutionary history of life on Earth, followed by a cross-check of our discoveries from the analysis with the system-environment interaction from Prigogine's dissipative structure (hereafter referred to as "living systems" ${ }^{1}$ ), Conditional Causality and Cyclic Adaptation have been discovered as the underlying principles that govern the incidents analyzed. Based on our discoveries, the authors would also like to argue that these two principles are also the fundamental principles underlying the creation and evolution of living dissipative structures.

This study focuses on the evolution of plants and animals, and demonstrates that the fundamental characteristic of living systems is "continuous adaptation to the environment", which means that, once appropriate environmental conditions are present, an orderly living system will emerge from a relatively disordered elemental state. When the environment undergoes further dramatic changes, the already formed living system will then evolve into a structurally and functionally more complex living system through phase transition.

The phase transition of a dissipative structure (which is also a living system) is based on the principle of Conditional Causality, which is a fundamental characteristic of any open system. When the environment of the living system in question changes, the living system will face death if it fails to adapt to the new environment. Therefore, the principle of Conditional Causality, as we can see, illustrates a process through which living systems adapt to the environment in a self-organized fashion.

\footnotetext{
${ }^{1}$ The scope of dissipative structures includes non-living natural systems (e.g., Benard cells, which are a physical thermodynamic phenomenon, or BZ (Belousov-Zhabotinsky) chemical clock, which is a chemical kinetic phenomena). However, given that this paper focuses its discussion on the topic of biological evolution, we have replaced "dissipative structures" with "living systems" for ease of discussion.
} 
The environment changes continuously with time. Therefore, any living system that adapts to the environment following Conditional Causality is bound to undergo Cyclic Adaptation in its life course. This Cyclic Adaptation happens because, over time, living systems that survive environmental changes presumably have undergone innumerable such changes without failure; on the other hand, living systems that fail to survive environmental changes will cease to exist. This kind of phase transition, which happens in response to environmental changes, is the so-called Cyclic Adaptation phenomenon. This phenomenon abides by the principle of Cyclic Adaptation and is consistent with the "Punctuated Equilibrium".

While this paper focuses on the evolutionary events of plants and animals on Earth, we believe that both Conditional Causality and Cyclic Adaptation, both of which are patterns of self-organization in living systems, are universal and also applicable to human organizational systems.

For example, Apple's rise to prominence came when the X86 microprocessor and BASIC had matured. Later, Steve Jobs adopted the concept of GUI, developed by Xerox, to launch the Mac PC, whose man-machine interface set itself as a brand new phenomenon. Later, inspired by MP3 and Walkman, Apple launched the iTunes and iPod. The iPhone, Apple's version of a smartphone, was brought forth after the iPod. The iPhone has revolutionized traditional design logics, thereby creating a dramatic new paradigm and sending shock waves through the market. This evolution of products is not only a manifestation of Conditional Causality but also serves to demonstrate Apple's Cyclic Adaptation as time passes.

Concerning human organizations, we believe that Conditional Causality and Cyclic Adaptation merit further application and research.

\subsection{Further Studies}

The evolution of high molecular compounds (such as proteins), RNA, DNA, prokaryotic cells, etc., is still full of unanswered questions or inconclusive hypotheses. The authors hope that, as these unanswered questions or inconclusive hypotheses become answered and established in the future, they will be subject to verification based on the two principles mentioned above.

The concept of "open system" initiated by von Bertalanffy (von Bertalanffy, 1968; Hammond, 2019; Ruzzeddu and Roblek, 2020). Subsequent studies of the open system concept have emphasized the importance of the interaction between the system in question and its environment, and the replacement of the traditional machinery view with an organic view (Harung et al., 1999; Styhre, 2002). However, the majority of such literature only advocate this kind of normative statement or declaration; few scholars have discussed the specific operational characteristics and implications of the organic relationship between the system and the environment.

We believe that, insofar as a living system is concerned, a theory of organic open systems should be able to clearly describe and explain the phenomena of creation, existence and evolution (including death), i.e., the regularity with which the entire life course follows. Buddha of the East has stated that a living system interacts with its environment according to a set of natural hierarchical order (Macy, 1991), and part of the concept of Conditional Causality in this paper was inspired by Buddhism as well. However, the results of the family of complex system theories (including dissipative structure, chaos, catastrophe, fractal, 
synergetic, etc.) have provided us with fundamental yet adequate materials with which to construct a new open organic system theory. These theories, in turn, shall serve as a sufficient basis for us to understand the macro and micro connotations of the living system's history.

This paper should be the beginning of such endeavor and the first to summarize the two basic principles mentioned above for discussion. We call for more experts in this field to combine the results of their respective branches of complex systems in a cross-sectional fashion, so as to construct a "new living system" theory we can apply to biology, ecology, human organization and society.

\section{References}

Bertalanffy, L. V. (1968). General System Theory: Foundations, Development, Applications. New York: George Braziller Inc.

Cheng, S. et al. (2019). Genomes of Subaerial Zygnematophyceae Provide Insights into Land Plant Evolution. Cell 179(5): 1057-1067.

Cramer, F. (1993). Chaos and Order: The Complex Structure of Living Systems Foreword by I Prigogine. New York: Wiley-VCH.

De Duve, C. (1995). Vital Dust: The Origin and Evolution of Life on Earth. New York: Basic Books.

Dole, M. (1965). The Natural History of Oxygen. The Journal of General Physiology 49(1): 5-27.

Dooley, K. J. \& Van de Ven, A. H. (1999). Explaining Complex Organizational Dynamics. Organization Science 10(3): 358-372.

Gould, N. \& Eldredge, N. (1972). Punctuated Equilibria: An Alternative to Phyletic Gradualism. In: Ayala, F. J. \& Avise J. C. (eds) Essential Readings in Evolutionary Biology, 82-115. San Francisco: Freeman Cooper.

Hammond, D. (2019). The Legacy of Ludwig von Bertalanffy and its Relevance for Our Time. Systems Research and Behavioral Science 36: 301- 307.

Harung, H. S. et al. (1999). Evolution of Organizations in the New Millennium. Leadership \& Organization Development Journal 20(4): 198-206.

Imachi, H., Nobu, M.K., Nakahara, N. et al., (2020). Isolation of an Archaeon at the ProkaryoteEukaryote Interface. Nature 577: 519-525

Lane, N. (2010). Life Ascending: The Ten Great Inventions of Evolution, Profile books. New York: W. W. Norton \& Company.

Macy, J. (1991). Mutual Causality in Buddhism and General Systems Theory: The Dharma of Natural Systems. New York: State University of New York Press.

Mao, C. K. (2018). Principles and Practices of Management - Integrating Decision Making and Implementation Perspectives. Taiwan: NCTU Press (In Chinese).

Margulis, L. et al. (2006). The Last Eukaryotic Common Ancestor (LECA): Acquisition of Cytoskeletal Motility from Aerotolerant Spirochetes in the Proterozoic Eon. Proceedings of the National Academy of Sciences 103(35): 13080-13085.

Margulis, L. and Sagan, D. (1997). Microcosmos: Four Billion Years of Microbial Evolution. California: Univ. of California Press. 
Melo AT. et al. (2020). Thinking (in) Complexity: (In) definitions and (mis) conceptions. Systems Research and Behavioral Science 37:154-169.

Prigogine, I. (1978). Time, Structure, and Fluctuations. Science 201(4358): 777-785.

Prigogine, I. and Nicolis, G. (1977). Self-organisation in Nonequilibrium Systems: Towards A Dynamics of Complexity. Bifurcation Analysis: Principles, Applications and Synthesis. In: Hazewinkel M., Jurkovich R., Paelinck J.H.P. (eds) Bifurcation Analysis. Dordrecht: Springer.

Prigogine, I. and Stengers, I. (1984 ). Order Out of Chaos: Man's New Dialogue with Nature. New York: Bantam Books.

Ruzzeddu, M., \& Roblek, V. (2020). Complexity Theories: A Historic Glance. World Complexity Science Academy Journal 1(1): 93-104.

Sagan, L. (1967). On the Origin of Mitosing Cells. Journal of Theoretical Biology 14(3): 225-226.

Styhre, A. (2002). Non-Linear Change in Organizations: Organization Change Management Informed by Complexity Theory. Leadership \& Organization Development Journal 23(6): 343-351.

Zachar, I. and Szathmáry, E. (2017). Breath-Giving Cooperation: Critical Review of Origin of Mitochondria Hypotheses. Biology Direct 12(1): 19.

Zachar, I. et al. (2018). Farming the Mitochondrial Ancestor as A Model of Endosymbiotic Establishment by Natural Selection. Proceedings of the National Academy of Sciences 115(7): 15041510.

\section{Acknowledgments}

The authors are grateful for the insightful suggestions provided by two anonymous reviewers.

\section{Conflicts of Interest}

The authors declare that there is no conflict of interest regarding the publication of this paper.

\section{Funding Statement}

The authors declare that there is no financial funding regarding the publication of this paper. 
This article is distributed under the terms of the Creative Commons Attribution 4.0

License (https://creativecommons. org/licenses/by/4.0/) which permits any use, reproduction and distribution of the work without further permission provided the original work is attributed as specified on the WCSA Journal by World Complexity Science Academy (https://www.wcsaglobal.org/ethics-policy/). 